\title{
Pattern based Residual Coding for H.264 Encoder*
}

\author{
Manoranjan Paul and Manzur Murshed \\ Gippsland School of Information Technology, Monash University, Churchill, Vic-3842, Australia \\ E-mail: \{Manoranjan.paul, Manzur.Murshed\}@infotech.monash.edu.au
}

\begin{abstract}
Video coding representing the moving region in Macroblock by pattern templates has already established its superiority over H.264 in low bit rate areas. In this approach, moving region covered by best matched pattern template is used to estimate the motion and encode the corresponding residual error. Due to the occlusion sometimes motion estimation of moving region using pattern template cannot reflect the true object movement. Moreover, uncovered moving region by pattern template may generate the wrong motion information. In this paper, we propose a novel pattern based residual coding covered by best matched template using full block motion vector instead of pattern covered moving region. Thus, unlike the present approach, it can avoid wrong motion vector due to the occlusion. Since this approach uses large block $(16 \times 16)$ motion estimation information, no computational overhead is needed compared to the H.264 video coding standard. The experimental results confirm that this new approach successfully reduces the bits stream significantly and as a result improves the rate-distortion performance compared to the existing pattern-based video coding as well as H.264 standard.
\end{abstract}

Keywords: Motion estimation, motion compensation, teleconferencing, video coding, pattern based coding.

\section{Introduction}

Video compression standards, such as H.263 [1] and MPEG-2 [2], are inefficient, while coding at low bit rate, due to their inability to exploit intra-block temporal redundancy which is almost static in successive frames. This results in all 256 residual error values being transmitted for motion estimation (ME) and motion compensation (MC) regardless of whether there are moving objects in the Macroblock (MB) or not. H.264/AVC has extended the block-based motion compensated coding idea by introducing variable-block size to approximate the shape of the moving objects

*This research was supported under the Australian Research Council's Discovery scheme (project number DP0666456). within the MB more accurately. Choosing larger partition sizes requires a relatively small number of bits for motion vectors and the type of partition but at the expense of a large number of bits for residual error. By contrast, choosing smaller partition sizes may result in a smaller number of bits for residual error at the expense of a larger number of bits for motion vectors and the type of partition. It can be easily observed that the possibility of choosing smaller partition sizes diminishes as the target bit rate is lowered. Consequently, coding efficiency improvement due to MB partitioning can no longer be realized for a low bit rate target as larger partition sizes have to be chosen in most cases to keep the bit-rate in check but at the expense of inferior shape approximation. Moreover, a moving object is not always approximate to the rectangular pattern which is assumed in H.264 variable block size ME and MC. The MPEG-4 [3] video standard exploits intra-block temporal redundancy, by dividing video frames into separate segments comprising a background and one or more moving objects. Paradoxically, it also depends on computationally expensive segmentation and shape coding and is not suitable for low processing devices, especially non-synthetic real-world objects.

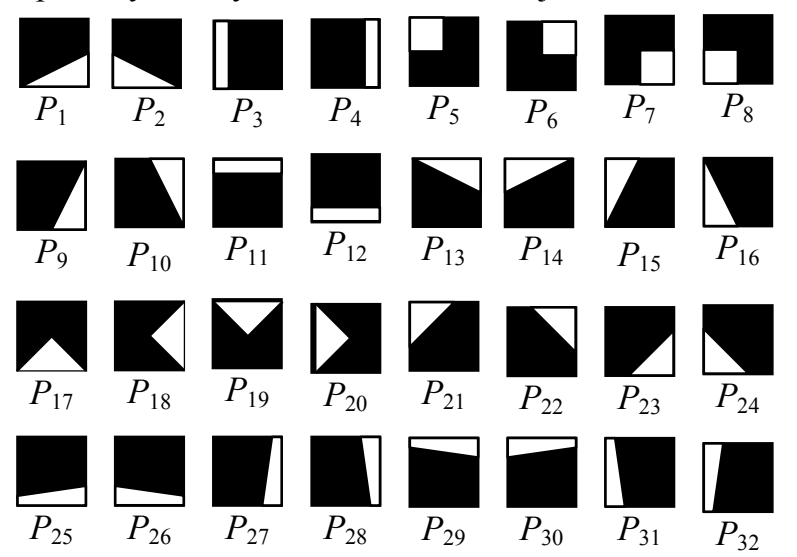

Figure 1: The pattern codebook of 32 regular shaped, 64-pixel patterns, defined in $16 \times 16$ blocks, where the white region represents 1 (motion) and black region represents 0 (no motion).

An alternative approach was proposed by Fukuhara et al. [5] who used four MB-partitioning patterns each comprising 128-pixels. ME and MC was carried out on all eight possible 128-pixel partitions of an $\mathrm{MB}$ and the pattern with the lowest prediction error was selected. Treating each MB, irrespective of its motion content resulted in a higher bit-rate being 
incurred for those MBs which contained only static background or had moving object(s), with little static background. In such cases, the motion vectors for both partitions were almost the same and so only one could be represented.

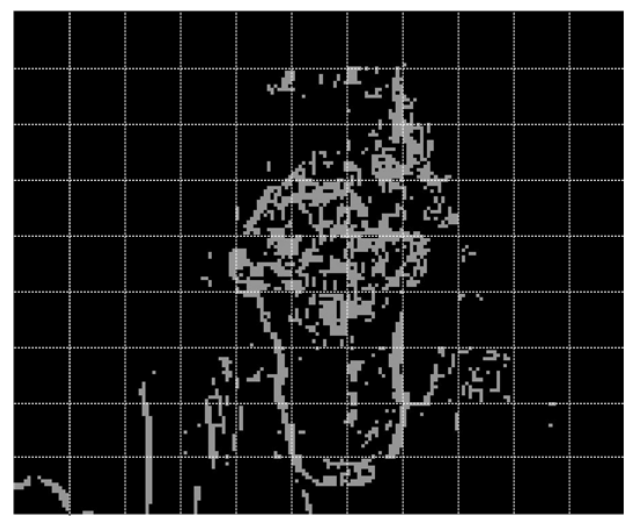

(a)

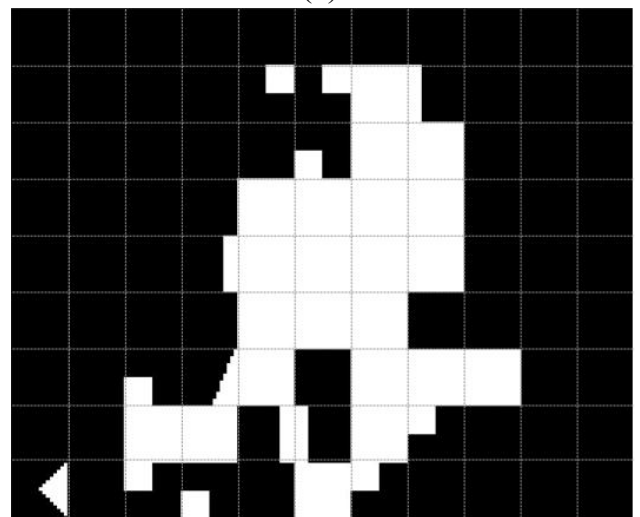

(b)

Figure 2: An example of pattern approximation for the Miss America standard video sequence, (a) detected moving region between frame one and two, and (b) results of pattern approximation.

To address the limitations of Fukuhara et al.'s approach, Wong el al. [8] and Paul et al. [7] exploited the idea of partitioning the MBs via a simplified segmentation process that again avoided handling the exact shape of the moving objects, so that popular MBbased motion estimation techniques could be applied. This pattern-based video coding (PVC) algorithm focused on the moving regions of the $\mathrm{MBs}$, through the use of a set of regular 64-pixel pattern templates (see Figure 1), from a pattern codebook (PC). Figure 2 shows an example of pattern-matching using 32 patterns for Miss America sequence. If in using some similarity measure [6], the moving region (MR) of an $\mathrm{MB}$ is well covered by a particular pattern, then the $\mathrm{MB}$ can be classified as region-active MB (RMB) and coded by considering only the 64 pixels of that pattern, with the remaining 192 pixels being skipped as static background. The remaining MBs are treated as H.264 did. Successful pattern matching can therefore, theoretically, have a maximum compression ratio of 4:1 for any MB. The actual achievable compression ratio will be lower due to object occlusion and the computing overheads for handling an additional MB type, pattern identification numbering and pattern matching errors. This approach is radically different from H.264 sub-blocking as ME and MC is carried out only for the selected patterns, thus keeping the computational complexity in check. Paul et al. [9] successfully included PVC as an extra mode in H.264 which outperforms the existing H.264 coder.

The main problem of existing pattern based video coding is that when an occlusion is occurred it cannot generate true motion using moving region covered by best matched pattern template. Moreover, uncovered moving region may change the motion estimation direction which may generate wrong motion vector. To overcome this problem we use whole macroblock as a unit for motion vector estimation. In this case we can easily use motion vector generated by $16 \times 16$ block so that no computational time is needed for pattern mode. Then we encode the part of the residual error which is best covered by a pattern template. The conventional pattern based video coding (PVC) from now on we termed as pre pattern based video coding (PrPVC) first find a pattern template which is suitable (based on some similarity measure) for the moving region of a macroblock. Then based on this moving region, motion estimation and compensation is processed. In the new approach from now on we termed it as post pattern based video coding (PoPVC), motion estimation and compensation is processed using the whole area of a macroblock but a part of residual error is encoded using best matched pattern template. Using this approach we significantly reduce the bitsstream and as a result we improve the rate-distortion performance compared to the existing pattern-based coding algorithm as well as H.264 video coding standard.

This paper is organized as follows: Section 2 describes existing pattern based video coding. Section 3 describes the proposed method. Section 4 analyses experimental set up and results. Section 5 concludes the paper.

\section{Pre pattern-based video coding (PrPVC)}

Let $C_{k}(x, y)$ and $R_{k}(x, y)$ denote the $k^{\text {th }} \mathrm{MB}$ of the current and reference frames, each of size $W$ pixels $\times H$ lines, respectively of a video sequence, where $0 \leq x, y \leq 15$ and $0 \leq k<W / 16 \times H / 16$. The moving region $M_{k}(x, y)$ of the $k^{\text {th }} \mathrm{MB}$ in the current frame is obtained as follows:

$$
M_{k}(x, y)=T\left(\left|C_{k}(x, y) \bullet B-R_{k}(x, y) \bullet B\right|\right)
$$

where $B$ is a $3 \times 3$ unit matrix for the morphological closing operation $\bullet[10]$, which is applied to reduce noise, and the thresholding function $T(v)=1$ if $v>2$ and 0 otherwise.

Let $|Q|_{\ell}$ be the total number of $\ell$ 's in the matrix $Q$. Similarity of a pattern $P_{n} \in \mathrm{PC}$ with the moving region in the $k^{\text {th }} \mathrm{MB}$ can be defined efficiently [6] as 


$$
S_{k, n}=\left|M_{k}\right|_{1}-\left|M_{k} \wedge P_{n}\right|_{1} .
$$

Clearly, higher the similarity lower will be the value of $S_{k, n}$. Obviously all MBs are not examined using pattern. The eligible MBs are called candidate RMB (CRMB) and defined as $8 \leq\left|M_{k}\right|_{1}<T_{S}+$ PatternSize, where $T_{S}$ and

PatternSize are the predefined similarity threshold and 64 respectively. The candidate CRMB is classified as an RMB and its moving region is represented by a pattern $P_{i}$ such that

$$
\left.P_{i}=\arg \min _{\forall P_{n} \in \mathrm{PC}}\left(S_{k, n}\right) \mid S_{k, n}<T_{S}\right)
$$

otherwise, the CRMB is classified as an AMB. For a given PC, an image sequence is coded using the PrPVC. To avoid more than four $4 \times 4$ block of DCT calculations for 64 residual error values per RMB, these values are rearranged into an $8 \times 8$ block. It avoids unnecessary DCT block transmission. A similar inverse procedure is performed during the decoding. After finding the bit rates and corresponding distortion using all modes including PrPVC, final decision is made based on the minimum Lagrangian cost function [11]. PrPVC classified $10 \%$ to $30 \%$ of macroblocks as RMB and improved the image quality $0.5 \mathrm{~dB}$ to $1.5 \mathrm{~dB}$ compared to the H.264 standard in low bit rate region.

To analysis the performance of PrPVC algorithm, we easily observe that the moving region covered by the best-matched pattern template provides relatively less bits with better image quality using one-fourth motion estimation computational time as the size of pattern is one-fourth of macroblock. On the contrary, uncovered moving region provides poor image quality. Since at low bit rate, a large scale distortion is occurred, thus image distortion due to the uncovered moving region is negligible compared to the high distortion in overall image. As a result, PrPVC algorithm outperforms the H.264 standard in terms of rate-distortion for very low bit rate range. In terms of computational complexity compared to the H.264, PrPVC (when used as an extra mode in H.264) requires little bit more computational time for selecting the best pattern for some moving region and encoding that moving region using pattern.

In the existing PrPVC algorithm there are two thresholds $v$ and $T s$ are used. The first threshold is used to generate the moving region if corresponding pixel intensity difference is more than ' 2 ' between current and reference macroblocks. Thus using this threshold PrPVC ignores some moving region where the corresponding pixel intensity difference is ' 1 ' or ' 2 '. The second threshold is used to classify a macroblock as RMB if the miss-matched region between bestmatched pattern and moving region is less $T_{s}$. Using this threshold the PrPVC ignores $T_{s}$ amount of moving region area for motion estimation. Thus, both thresholds may generate wrong motion vector and then large residual error. Moreover, significant amount of computational complexity can be reduced if we can use motion vector which is already generated by other modes of H.264 for pattern mode.

\section{Post pattern-based video coding (PoPVC)}

Normally motion vector is calculated in PrPVC using pattern covered moving region instead of whole block to reduce the computational time. But from the experimental results we observed that motion estimation using whole block instead of pattern covered moving region provides better results. When pattern based video coding is used as an extra mode in H.264, we can easily use motion vector generated by $16 \times 16$. Thus no computational time is needed for motion estimation. In PoPVC algorithm we generate a binary matrix from the residual error between current block and motion estimated reference block instead of co-located reference block used in PrPVC. Then we find the best matched pattern among the 32 patterns codebook (see Figure 1) using the similarity measure (2). If the similarity metric is within a pre-defined threshold, then we classify it as a pattern representable residual error. Based on the minimum Lagrangian cost function final decision is made for a macroblock. As motion vector and residual error are calculated in PoPVC using whole block, the rate-distortion performance will be improved. Moreover, calculating motion vector does not require any computational time as they are already generated by other mode and selecting the best matched pattern for residual error does not change any order of computational complexity compared to the H.264 coding standard.

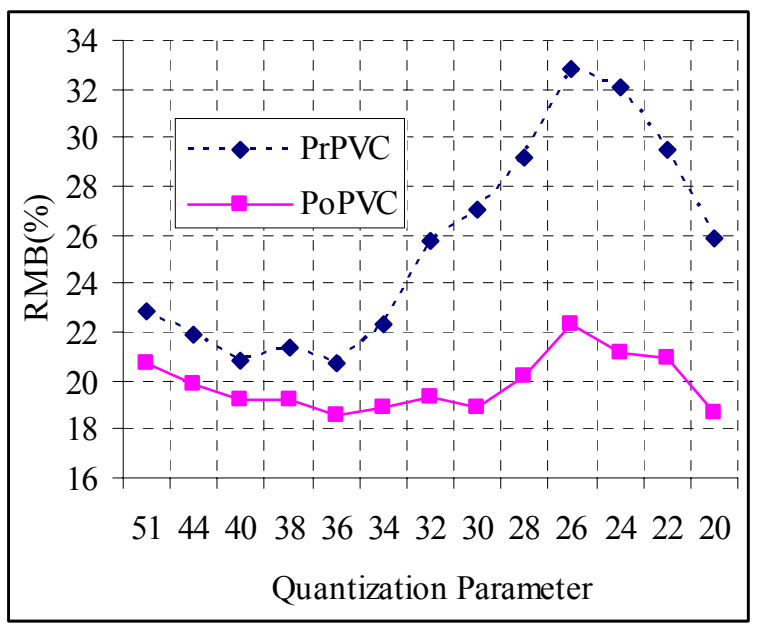

Figure 3: Percentage of RMBs using both PrPVC and PoPVC algorithms for Miss America standard video sequence.

For the same similarity threshold in both PrPVC and PoPVC algorithms, the number of RMBs is varied (see Figure 3). In both cases percentages of macroblocks encoded using large blocks is almost same but this is not the case for small blocks $(8 \times 8,8 \times 4,4 \times 84 \times 4)$. The percentages of small modes in PoPVC are relatively 
more compared to that of PrPVC which indicate the better image quality.

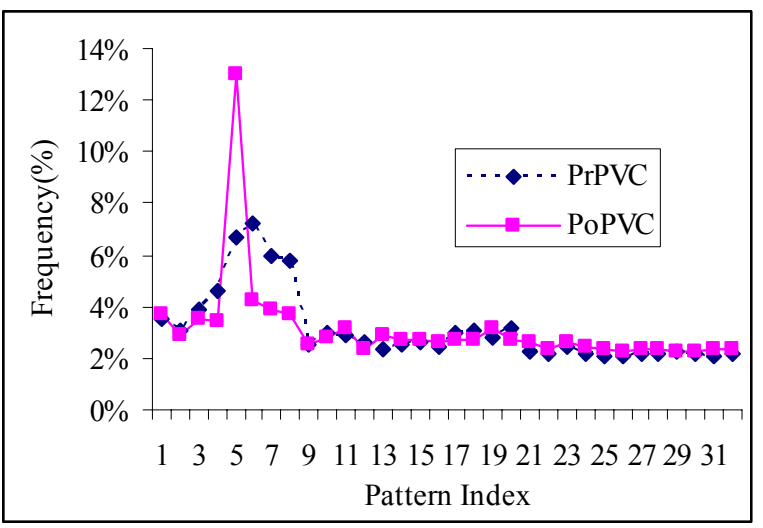

Figure 4: Pattern frequency using both PrPVC and PoPVC for Miss America video sequence when QP $=\mathbf{2 0}$.

The distributions of pattern frequency based on the number of RMBs classified using that pattern are different when both PrPVC and PoPVC algorithms are used (see Figure 4). From this figure we can easily observed that most frequent patterns are within first eight patterns in both cases. This figure also indicates that different pattern set may be suitable for PoPVC algorithm which yet to be investigated.

For PoPVC, existing H.264 encoder and decoder architecture needs to be slightly modified. Pattern codebook needs to be added in encoder and decoder so that pattern shape can be identified from the index of the pattern which is included in the header of the macroblock. For 32 patterns, 5 bits index is included in addition to the macroblock type of existing syntax.

\section{Simulation Results}

We have implemented our proposed algorithms based on the Baseline profile of H.264/AVC with full search motion estimation, 15 group of picture, up to Half-Pel accuracy [12] for number of standard and non-standard QCIF [13] video sequences with 15 frames per second (fps). In both PrPVC and PoPVC algorithms used same PC, similarity metric, and similarity threshold. To keep consistence in various bit rates we use different similarity threshold which is a function of Quantization Parameter (QP). In the experimental results we observe that $T_{s}=2 \mathrm{QP} / 3$ provides consistence rate-distortion performance with that of H.264 standard. The more the value the more is the uncovered moving region or residual error which degrades the image quality but reduces the size of the bit stream.

Figure 5 shows the final rate-distortion performance using H.264, PrPVC, and PoPVC algorithms for four standard video sequences. PoPVC algorithm performed better compared to the other two algorithms. Foreman sequence has low number of RMBs thus the performance of PoPVC is very close to the other two algorithms.
We didn't investigate yet whether the same set of patterns is suitable for both PrPVC and PoPVC. Generation of optimal number of patterns is another difficult issue yet to be investigated. Currently we use 64-pixel pattern size. Further research is needed to investigate whether different pattern size would improve the rate-distortion performance.

\section{Conclusions}

In this paper a novel pattern based residual coding is proposed which can overcome the wrong motion vector generation by the existing pattern based video coding especially when there is an object occlusion in video sequence. This technique successfully used already generated motion vector by other mode of H.264 standard so that no motion estimation overhead is needed. Pattern template is used to identify the portion of residual error which needs to be encoded. Since the pattern size is one-fourth of the macroblock size, thus, successfully matched residual error with a pattern can save three fourth of bits stream. The experimental results reveal that this new technique improved the rate-distortion performance compared to the existing pattern based video coding as well as H.264 video coding standard without adding any computational cost.

\section{References}

[1] ITU-T Recommendation H.263, "Video coding for low bit-rate communication," Version 2, 1998.

[2] ISO/IEC 13818, MPEG-2 International Standard, 1995.

[3] ISO/IEC N4030, MPEG-4 International Standard, 2001.

[4] ITU-T Rec. H.264/ISO/IEC 14496-10 AVC. Joint Video Team (JVT) of ISO MPEG and ITU-T VCEG, JVT-G050, 2003.

[5] Fukuhara, T., K. Asai, and T. Murakami, "Very low bit-rate video coding with block partitioning and adaptive selection of two time-differential frame memories," IEEE Trans. Circuits and Systems for Video Technology, vol. 7, 212-220, 1997.

[6] Paul, M., M. Murshed and L. Dooley, "A new efficient similarity metric generic computation strategy for pattern-based very low bit-rate video coding," Proc. of IEEE Int. Conference on Acoustics, Speech, and Signal Proc., vol. 3, 165-168, 2004.

[7] Paul, M., M. Murshed, and L. Dooley, "A realtime pattern selection algorithm for very low bit-rate video coding using relevance and similarity metrics," IEEE Trans.Circuits and Syst on Video Technology, vol. 15(6), 753-761, June, 2005.

[8] Wong, K.-W., K. -M. Lam, and W. -C. Siu, "An efficient low bit-rate video-coding algorithm focusing on moving regions," IEEE Trans. on Circuits and Systems for Video Technology, vol. 11(10), 1128-1134, 2001. 
[9] Paul, M. and M. Murshed, "Efficient H.264/AVC video encoder where Pattern is Used as Extra Mode for Wide Range of Video Coding ," International Multimedia Modeling Conference (MMM-07) Lecture Notes in Computer Science, Springer-Verlag, Heidelberg.

[10]Maragos, P., "Tutorial on advances in morphological image processing and analysis," Opt. Eng., vol. 26(7), 623-632, 1987.

[11] Weigrand, T., H. Schwarz, A. Joch, and F. Kossentini, "Rate-contrained coder control and
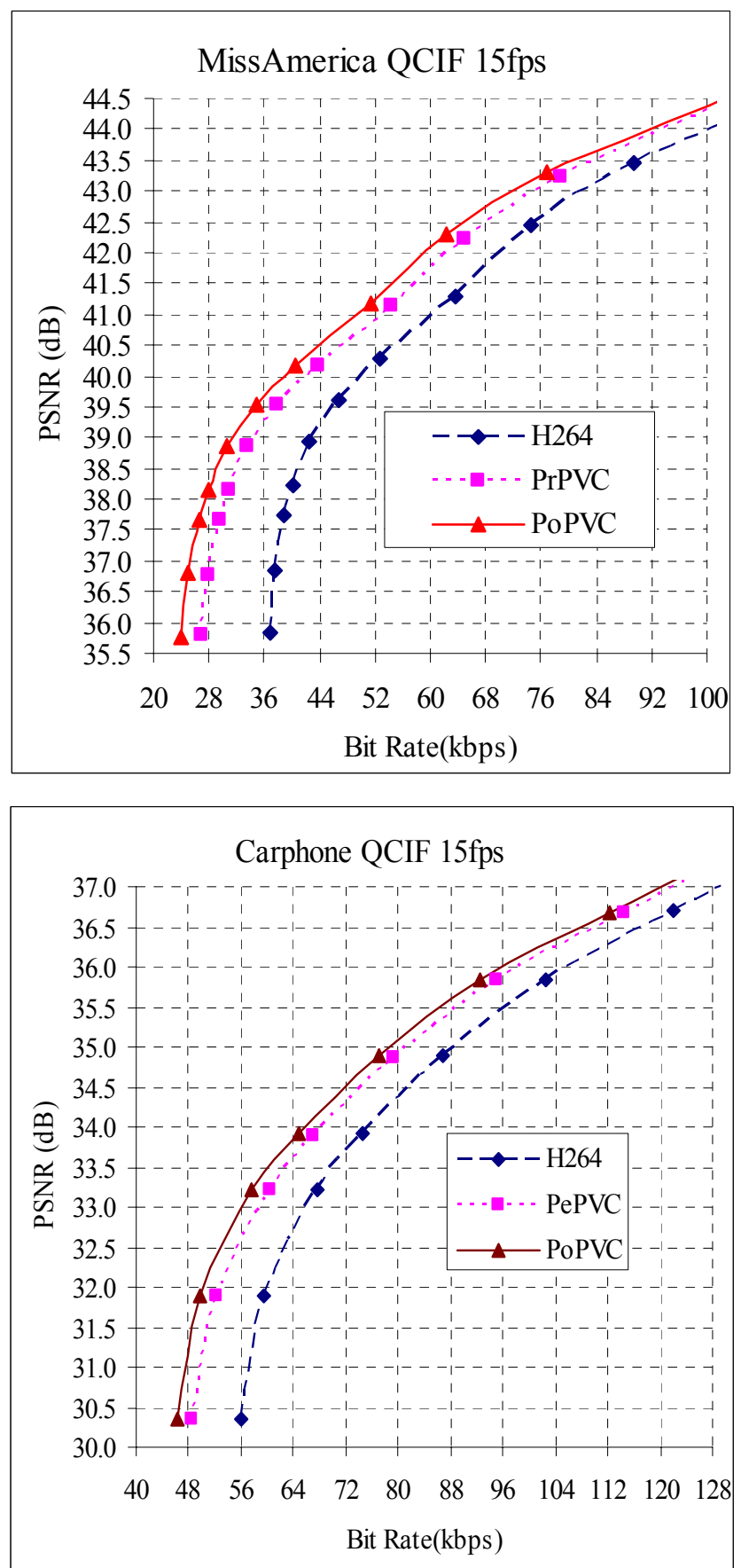

comparison of video coding standards," IEEE Trans. on Circuits and Systems on Video Technology, 13 (7), 688-702, 2003.

[12] Shi, Y.Q. and H. Sun, Image and Video Compression for Multimedia Engineering Fundamentals, Algorithms, and Standards, CRC Press, 1999.

[13] Richardson, I. E. G., "H.264 and MPEG-4 video compression," Wiley Press, 2003.
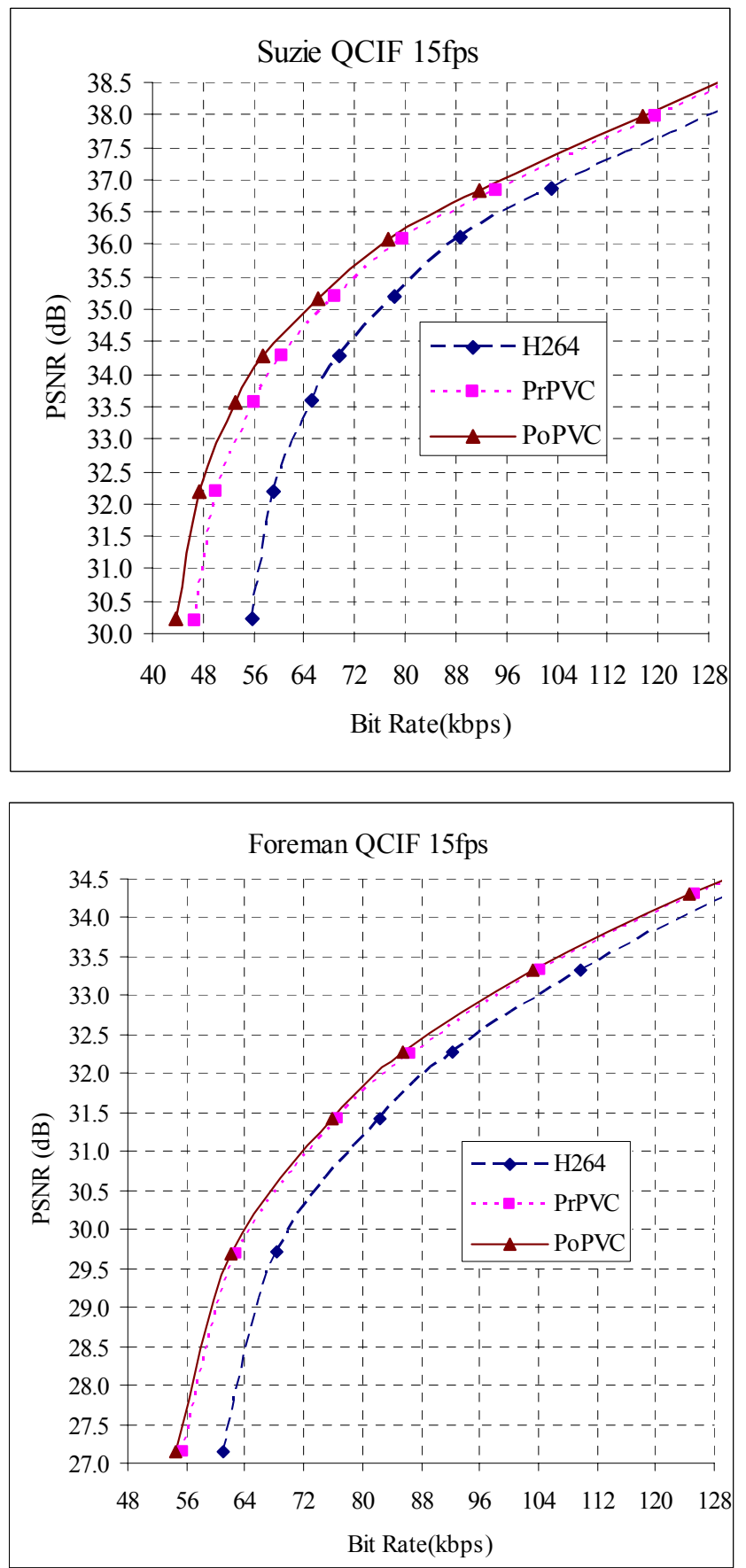

Figure 5: Rate-Distortion curves for four standard video sequences namely Miss America, Sussie, Carphone, and Foreman of QCIF with 15 frames per second. 